\title{
Reflets
}

Revue ontaroise d'intervention sociale et communautaire

\section{Développement économique communautaire en Ontario français : tour d'horizon et questions}

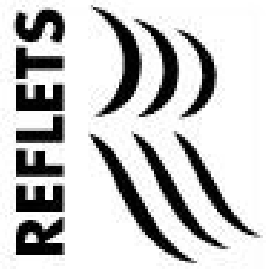

\section{Rachid Bagaoui, Donald Dennie et Nérée St-Amand}

Volume 5, numéro 1, printemps 1999

Pratiques et développement économique communautaire

URI : https://id.erudit.org/iderudit/026246ar

DOI : https://doi.org/10.7202/026246ar

Aller au sommaire du numéro

Éditeur(s)

Reflets : Revue ontaroise d'intervention sociale et communautaire

ISSN

1203-4576 (imprimé)

1712-8498 (numérique)

Découvrir la revue

Citer cet article

Bagaoui, R., Dennie, D. \& St-Amand, N. (1999). Développement économique communautaire en Ontario français : tour d'horizon et questions. Reflets, 5(1),

8-15. https://doi.org/10.7202/026246ar

Tous droits réservés (C) Reflets : Revue ontaroise d'intervention sociale et communautaire, 1999
Ce document est protégé par la loi sur le droit d'auteur. L'utilisation des services d’Érudit (y compris la reproduction) est assujettie à sa politique d'utilisation que vous pouvez consulter en ligne.

https://apropos.erudit.org/fr/usagers/politique-dutilisation/ 


\section{Développement économique communautaire en Ontario français: tour d'horizon et questions}

\section{Rachid Bagaoui et Donald Dennie}

professeurs, Université Laurentienne

Nérée St-Amand, professeur, Université d'Ottawa

Lorraine Guay (Klein et al. 1997) note «qu'au sein du mouvement communautaire, plusieurs ont l'impression que la question de l'économie sociale leur est tombée sur la tête... comme le ciel sur ces Gaulois célèbres. Plusieurs se demandent avec raison ce qu'est cette nouvelle bébite» (135). S'il est vrai que l'économie sociale est loin d'être une nouvelle réalité, il faut reconnaître que la morosité du contexte social depuis une quinzaine d'années l'a propulsée sur la scène publique. Sa médiatisation et le débat qu'elle suscite depuis lors laissent peu de gens indifférents, tant dans le domaine des politiques sociales et de la recherche que de l'intervention.

Inspirés par ce vaste mouvement qui propose diverses alternatives à l'économie néolibérale, les auteurs de ce numéro se sont posé des questions sur la nature des liens entre économie et social dans un contexte particulièrement difficile pour tout projet qui, relevant du local, interpelle à la fois le politique et l'économique.

Développement local, développement économique communautaire, économie solidaire, services de proximité, économie sociale, économie alternative, autant de termes qui ont retenu 
notre intérêt lorsque nous avons proposé un numéro qui soulèverait la question de la place du développement économique communautaire (DÉC) dans la communauté franco-ontarienne. Nous reconnaissions au départ que les grands débats entourant l'économie sociale ont eu peu d'échos en Ontario, étant donné, en particulier, la collusion entre les forces économiques et politiques au pouvoir et l'agenda d'un gouvernement qui laisse peu de place, et encore moins de reconnaissance, aux initiatives porteuses de solidarités. Comme Boivin et Fortier (1998), nous sommes d'avis que «la justice sociale est une chose trop importante pour la laisser entre les mains de ceux qui n'ont que le silence et l'espoir à proposer» (9).

Quelles formes d'économie permettraient de concilier à la fois les besoins d'un développement durable et la participation réelle des populations de plus en plus marginalisées? Qui sont les acteurs et actrices d'autres formes de développement présentement à l'oeuvre en Ontario français? Quelles pratiques d'engagement solidaire surgissent de ces initiatives? Quelles stratégies collectives inspirent ces initiatives de solidarité? Pouvons-nous concevoir autrement la mondialisation en nous inscrivant dans le contexte d'un arrimage local-mondial et d'une vision planétaire du développement? Comme on l'a souligné aux Rencontres mondiales $d u$ développement local, nous sommes d'avis que «nos sociétés ont besoin de références éthiques et politiques pour ouvrir de nouveaux horizons et pour bâtir une démocratie au service des hommes et des femmes. Nous vivons dans un monde où de partout surgissent des initiatives, des mouvements de citoyens et de citoyennes déterminés à changer le visage de la planète, en conciliant ouverture sur le monde et satisfaction locale des besoins» (1-2).

Alors qu'émergent de nombreuses pratiques qui remettent en question les principes économiques traditionnels, que signifie, pour l'Ontario français, le terme DÉC? Jusqu'à quel point ce concept et les pratiques qui en découlent sont-ils présents actuellement? Les textes présentés indiquent clairement que le terme économie sociale est à peu près absent du vocabulaire de la réalité du terrain, les intervenantes et intervenants utilisant davantage celui de développement économique communautaire. 
Ensuite, les initiatives qui émergent, comme le soulignent Rachid Bagaoui et Donald Dennie dans leur article, sont dictées par une philosophie pragmatique : le DÉC semble davantage un outil, un moyen pour répondre à des problèmes de l'heure. Dans ce contexte, la réalité du DÉC se déploie-t-elle en marge du débat sur l'orientation de notre société? Ou encore les pratiques communautaires dont il sera question sont-elles porteuses d'un mouvement de remise en question des forces qui consomment les liens entre les humains et la nature? Voilà certaines des questions auxquelles nous vous convions à la lecture de ce numéro.

Voyons d'abord la définition qu'on accole au terme de DÉC.Si on se base sur la réalité du terrain, sur le langage adopté par les intervenants et les intervenantes, la définition la plus utilisée par les acteurs et actrices du DÉC en Ontario est celle de Jean-Marc Fontan (1994), pour qui le DÉC représente une stratégie basée sur:

- la prise en main par une population de son propre développement;

- la volonté de s'attaquer à toutes les formes de marginalisation socio-économique rencontrées, tant par les groupes concernés que dans les territoires touchés;

- l'importance accordée aux aspects sociaux et économiques dans toute initiative de développement.

Certes, ce numéro nous démontre que depuis quelques années, plusieurs organismes et ressources communautaires investissent de plus en plus, et de façon différente, le champ de l'économique. Plusieurs contributions qui ont été réunies visent à jeter un éclairage théorique et présenter un matériel empirique qui permettront d'alimenter la réflexion et la pratique des personnes concernées par ces initiatives. Repassons brièvement les grands thèmes qui s'en dégagent.

Le numéro s'ouvre sur une entrevue avec Éthel Côté, directrice générale du Conseil de la coopération de l'Ontario. Dans ce texte, madame Côté nous propose sa définition et sa vision du développement économique communautaire; par la suite, elle énumère des exemples concrets de DÉC en Ontario français. Insistant sur la primauté de la prise en charge locale et la création d'emplois sur la recherche de profits, elle souligne par la suite le 
rôle capital du Conseil de coopération de l'Ontario pour réunir les forces vives du DÉC en Ontario français.

L'article de Paul Leduc-Browne discute de la place de l'économie sociale dans la logique d'une économie néolibérale. Soulignant à la fois l'ambiguité et le potentiel de l'économie sociale, il passe en revue différents travaux sur la question tout en les situant dans le débat sur l'économie solidaire. S'agit-il de stratégies de récupération par l'État ou plutôt d'un modèle de développement économique au service du développement social? La question reste posée, mais l'auteur souligne que l'économie ne pourra jamais réellement devenir solidaire, même si l'approche est "séduisante», tant et aussi longtemps qu'elle restera locale. Il interpelle les gouvernements à reconnaitre leurs responsabilités plutôt que de les refiler à des groupes populaires, les réduisant ainsi «au statut de sous-traitants dans le nouvel ordre néolibéral».

Les activités de DÉC sont nombreuses et variées. Ce sont des initiatives d'appui et de création d'entreprises, la plupart du temps dans le domaine des services, les centres culturels, la construction des coopératives d'habitation, etc. Les activités du DÉC existent aussi sous diverses formes: coopératives, entreprises communautaires, organisation économique communautaire, etc. La plupart de ces initiatives, comme le souligne David Welch, ne sont pas nouvelles: certaines organisations comme les coopératives, et particulièrement les caisses populaires, remontent à très loin; les autres sont nées dans les années 1980 et 1990, souvent en réaction à une crise comme la fermeture d'une usine ou à une problématique comme la pauvreté des femmes ou le chômage chez les jeunes. Tout en nous brossant un tableau des origines historiques du développement économique communautaire en Ontario français, cet article fait donc ressortir certaines des forces de l'Ontario français qui sont en mesure de déboucher - et qui de fait débouchent — sur des activités de DÉC.

L'article de Rachid Bagaoui et Donald Dennie problématise la question du DÉC en Ontario. Ces auteurs illustrent, à l'aide de multiples exemples, tant historiques que contemporains, tirés de leurs recherches actuelles sur le DÉC, la transformation actuelle du mouvement communautaire et l'émergence du DÉC. Tout 
comme David Welch, ils montrent que les initiatives du DÉC viennent de loin - comme question sociale, cependant, le DÉC remonte au début des années 1990 (Klein et al 1997). Bien que le mouvement communautaire vive aujourd'hui des difficultés de toutes sortes, liées en particulier au contexte néolibéral actuel, mais aussi à l'organisation interne du mouvement, la lutte des intervenants et intervenantes pour se renouveler est réelle et dynamique. Pour ces chercheurs, les initiatives du DÉC qui émergent en Ontario sont teintées par le pragmatisme: elles se veulent un outil de développement et ne s'inscrivent pas dans un projet de société anti-néolibéral clairement défini.

L'article signé par Jacinthe Michaud démontre que les femmes aux prises avec des conditions de pauvreté inventent et développent des stratégies de résistance pour faire face à la précarité. En se basant sur ces initiatives, l'auteure propose des pistes de réflexion au sujet du travail obligatoire des femmes qui luttent pour leur survie. Pour ces femmes, coincées entre des politiques sociales punitives et des projets inventifs, la question de cette auteure reste posée: "Plutôt que de gérer la richesse, l'économie sociale ne serait-elle pas plutôt une façon de gérer la pauvreté?»

La section «Des pratiques à notre image» présente deux projets qui nous invitent à appliquer les concepts de DÉC à deux clientèles particulières: les femmes aux prises avec la violence et les survivantes et survivants de la psychiatrie.

Paula Haapenen, du Centre des femmes francophones du Nord-Ouest de l'Ontario (CFFNOO) nous démontre qu'en travaillant dans le domaine de la violence faite aux femmes, on peut intégrer la dimension économique, proposer des microentreprises et, ce faisant, engager les usagères concernées dans leur propre développement. Insistant sur le fait que le tiers-secteur ne devrait pas demeurer à l'écart de l'économie, elle propose des projets mettant l'accent sur la cohésion sociale, la création de liens de solidarité et l'autonomie financière des femmes francoontariennes. 
Kathryn Church, par ailleurs, propose une réflexion basée sur dix ans de pratique de DÉC avec des personnes survivantes de la psychiatrie. Confrontées à la pauvreté chronique et l'exclusion sociale, ces personnes se sont mobilisées et ont créé des ressources inspirantes à plusieurs niveaux. Malgré l'hétérogénéité (croyances, aptitudes, forces, etc.) de cette communauté, elle démontre que l'Ontario Council of Alternative Businesses a réussi à créer et maintenir de nombreux projets qui mettent en évidence les ressources et la débrouillardise des personnes. Les cinq principes qu'elle en dégage dénotent à la fois l'immense potentiel des personnes et les défis de travailler avec des gens marqués par un système psychiatrique opprimant.

Dans la section "Aux quatre coins de la province», nous avons réuni quelques monographies du DÉC. La contribution de Donald Dennie porte sur la mobilisation de la population de la nouvelle municipalité de Rivière-des-Français, située à quelque 80 kilomètres au sud de Sudbury, en vue de trouver des solutions aux problèmes suivants qui affligent la région : les jeunes doivent laisser la région pour se trouver des emplois; les aînés partent aussi parce qu'il n'y a pas de résidence pour personnes âgées ni de services de santé permanents; l'agriculture périclite depuis de nombreuses années et le plus gros employeur de la région, la compagnie forestière Tembec, a décidé de ne pas reconstruire une scierie détruite par les flammes, mettant ainsi au chômage 80 travailleurs. La région ne compte plus d'industrie majeure. Devant l'ampleur de ces problèmes, des citoyens et des citoyennes se prennent en main en créant une coopérative de santé.

Le texte de Natalie Dupont porte sur la contribution d'une caisse populaire au développement communautaire. L'auteure démontre que la caisse est très active dans le développement de sa région en soutenant financièrement et techniquement les associations qui y oeuvrent dans le domaine du développement socio-économique et culturel. L'enquête menée auprès d'une trentaine de ces associations montre que leurs membres sont très satisfaits de l'apport de leur caisse, même si beaucoup de travail reste à faire.

La contribution de Jean-Gilles Lemieux porte sur la coopérative Boréal. Il examine le fonctionnement organisationnel 
de celle-ci, la satisfaction des employés et des employées ainsi que la place qu'occupent la culture et la langue française au sein de cette coopérative. L'auteur fait ressortir également le rôle que joue la coopérative auprès des apprenants et apprenantes du collège pour leur permettre de participer pleinement à la vie collégiale.

Dans le même esprit, l'article de Mufaume Kihumbi présente La Maison Verte. Établie à Hearst depuis 1982, cette entreprise communautaire a pour objectif, depuis ses débuts, de procurer des emplois pour des femmes et de s'enraciner dans sa communauté pour la faire bénéficier de ses activités et retombées économiques. L'auteur examine le fonctionnement organisationnel de cette entreprise, la place des employées et sa contribution au développement de sa région.

Soulignons enfin une nouveauté dans REFLETS. La bourse RIFAS (Regroupement des intervenantes et intervenants contre l'abus sexuel) est octroyée, depuis 1998, à un ou une étudiante de l'École de service social de l'Université d'Ottawa et vise à souligner l'excellence d'une recherche dans le domaine de la violence. À compter de maintenant, REFLETS est heureux de publier, une fois l'an, l'article de la récipiendaire de cette bourse. Tout en félicitant Marise Denault pour son excellent travail, nous voulons reconnaître l'originalité de son sujet d'étude, qui porte sur le harcèlement criminel suite à une rupture conjugale.

Nous espérons que ce numéro, en plus de reconnaitre certaines des pratiques actuelles, des questionnements et des recherches qui ont cours sur le sujet du DÉC, servira à alimenter les réflexions, les recherches et les pratiques en émergence. Il ne s'agit que d'un début, certes, mais les bases sont là pour le renouvellement des pratiques sociales axées sur des valeurs de solidarité, de dynamisme et de conscience des nuances et des enjeux du DÉC en Ontario français. 


\section{Bibliographie}

BOIVIN, Louise et Mark FORTIER (1998). L'économie sociale, l'avenir d'une illusion, Fides.

COLLOQUE INTERNATIONAL SUR LE DÉVELOPPEMENT ÉCONOMIQUE

COMMUNAUTAIRE (1992). Compte-rendu, Toronto, La Direction du développement communautaire du ministère des Affaires municipales.

FONTAN, J. M., (1994). «Le développement économique communautaire québécois : éléments de synthèse et point de vue critique», Revue internationale d'action communautaire, vol. 32, no 72,115126.

KLEIN,Juan-Luis, Pierre-André TREMBLAY et Hugues DIONNE (s. la dir. de) (1997). Au-delà du néolibéralisme: quel rôle pour les mouvement sociaux?, Presses de l'Université du Québec, 135-153.

RENCONTRES MONDIALES DU DÉVELOPPEMENT LOCAL (1998). Déclaration finale, Sherbrooke. 